\title{
Anycast Technique to Increase the Life Time of Wireless Sensor Network
}

\author{
Rahul C. Salunkhe \\ SITS,Narhe \\ Pune
}

\author{
R. H. Borhade \\ Assistance Professor \\ SKNCOE, Vadgaon,
}

\begin{abstract}
Wireless sensor network is collection of sensor nodes and one sink node. Sensor nodes sense the signals and forward to the sink node. In event driven wireless sensor network where some event is happened that event is sense by one of the sensor node and forward to sink node. Sensor nodes are small in size so the energy capacity of sensor node is very less. In the event driven wireless sensor network energy is consumed when radios are on waiting for packet to arrive. In most of the Event-Driven wireless sensor network Sleep Wake-Up scheduling is used where most of the time sensor node is in sleep mode so that the energy is saved but one drawback of this sleep wake up scheduling is that it introduces delay in the network. So it is very important that to save the energy so that life time of sensor node is maximize for this "Anycat Packet" Forwarding scheme is used where each node forwards the packet to the first neighboring node that wake up among multiple nodes. In sleep wake-up scheduling energy consumed by sensor nodes are depends on the wake up rate of the node. If the wake - up rate of node having less energy is more then that node not alive more time. This paper gives the details of how anycast forwarding scheme is used along with Sleep Wake up scheduling to balance the wake up rates of all sensor nodes so that the lifetime of WSN is increase.
\end{abstract}

\section{Keywords}

WSN, Anycast technique, Sleep -Wake up scheduling, Sensor node, Asynchronous.

\section{INTRODUCTION}

As a new technology in wireless sensor network is evolved so that the capability of remotely sense the environment is increase. Such a system are placed in the area where there is hard to reach. So these system should work for long duration. This work is concentrate on event driven asynchronous sensor networks where event occur rarely. This type of network is used in the environmental monitoring, intrusion detection etc. In such a event driven asynchronous sensor network there are four source of energy consumption.

1 To keep the radios on

2 To Transmission and Reception of control packets.

3 .To keep sensor on.

4. To data transmission and Reception.

This work is concentrate on asynchronous sleep wake-up scheduling protocols where each node wakes up independently of neighboring node in order to save the energy also concentrate on the balancing of the energy of each sensor nodes in the network so that the life time of the system will maximize.

The energy balancing is done with the help of asynchronous sleep wake up scheduling and Anycast forwarding schemes. In this we change the wake up rate of sensor nodes with the parameter as the remaining energy of that particular sensor node. As we modify the wake -up rate of sensor nodes the life of that sensor node is maximize so the life of network is also maximize.

The rest of the paper is organized as follows. In section II mention the details of Anycast protocol. Section III mention the detail System Model which is used to Maximize the life time of WSN and in section IV conclusion

\section{ANYCAST PROTOCOL}

Anycast is use of routing and addressing policies to find the most efficient path between source and multiple destination receivers. Layer 3 of routing is used to find out the significant target so that the packet is transferred
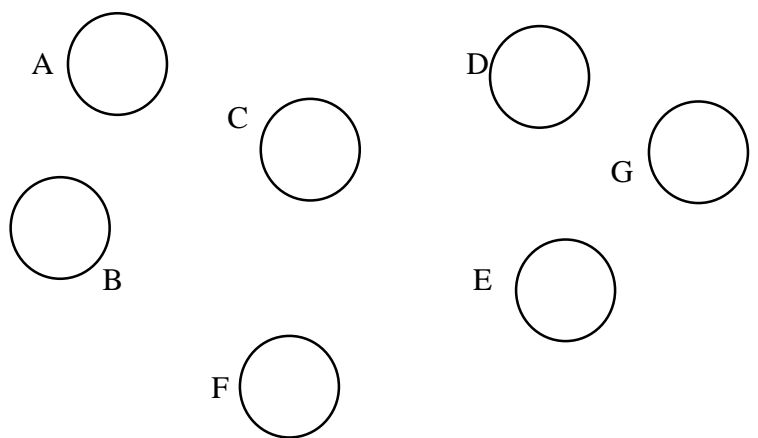

$\mathrm{E}$

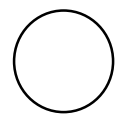

Fig. 1 WSN of N Nodes

Forwarding set of $A$ is $\{B, C\}$ means the node $A$ can transmit the packet to either $\mathrm{B}$ or $\mathrm{C}$ depends on the wake-up position of $\mathrm{B}$ and $\mathrm{C}$.

\section{SYSTEM MODEL}
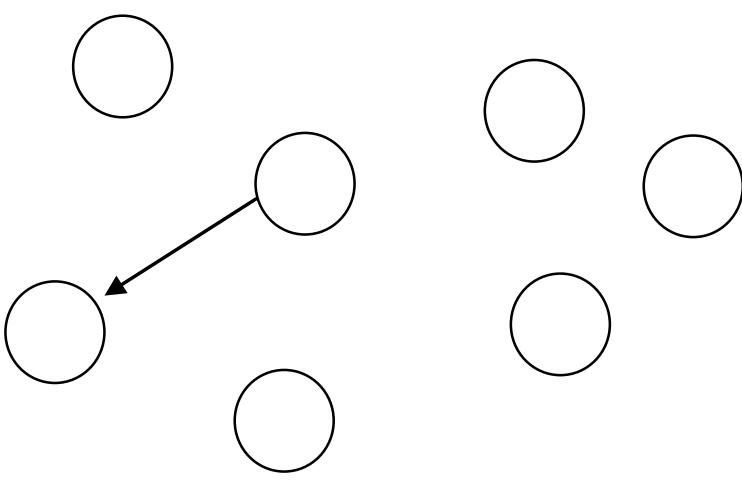

Fig. 2 WSN of N Nodes 
In Anycast forwarding technique consists of forwarding set which is nothing but the set of nodes to which the packet will forward among all the neighbors.

In traditional packet forwarding scheme, every node has designed next hop to which packet should forward. If that node is destroy then that packet is not forward to destination. While in Anycast forwarding scheme that packet is transferred to that node which is in Wake up state now.

Suppose From the figure 2 if the node $i$ has packet to transmit with The help of Anycast forwarding scheme and asynchronous sleep wake up scheduling the packet is forward to node $\mathrm{j}$.

\subsection{Sleep wake up Scheduling policy:}

Scheduling policy is used to find out how to forward the packet from source to destination. Sleep Wake up scheduling is used to find out when the nodes are wake-up. This sleep wake up scheduling is used to increase the lifetime of sensor nodes. Asynchronous sleep wake up each node wake up independently of neighboring nodes in order to save energy.

Sleep wake - up scheduling is fully characterized by

Wake-Up Rate:- Frequency the node wake up

Wake-Up pattern:- The Sharing of wake up intervals.

Wake Up Rate:-

The wake up rate of node is the average of times that node wake up. Lower the Wake -Up rates result in less energy consumption.

$$
\text { Wake - up rates } \alpha \frac{1}{\text { Life time of node }}
$$

\subsection{Energy balance using Asynchronous anycast protocol}

\subsubsection{Configuration Phase}

Configuration phase means initial phase where every node decides the wake up interval and pattern.

\section{2..2 Operation Phase}

When node sense the data and wants to send it, then it broadcast the ready to send signal along with update interval and remaining energy of self.

Anycast packet forwarding scheme is used for the communication.

Wake up rate of the receiving node which is intermediate node is changed according to the wake up rate of sender and remaining energy of sender and wake up rate of self and remaining energy of self.

If node $\mathrm{j}$ received RTS from node $\mathrm{i}$ with wake up interval Wi and remaining energy $\mathrm{REi}$

Then the modified wake up rate for node $\mathrm{j}$ is calculated by following method.

Wjnew $=W i+\mu 1 \Delta W i$ Eq-1
Where

$\mu 1$ - Constant deciding smothers of change

$\Delta \mathrm{Wi}$ - $\quad$ Change in Wi

$\frac{N e w \text { wake }- \text { up raete for } j}{\text { Wake-up rate for } i}=\frac{R E j}{R E i * W i}$

$\Delta \mathrm{W}_{\mathrm{J}(\text { Change in wake up rate })}=$ Wake up-old - Wake up-new

$\Delta \mathrm{WJ}$ (Change in wake up rate) $=\quad W j=R E j * W i$

$\Delta W j=W a k e-u p J-\frac{R E j * W i}{R E i} \quad \mathrm{Eq}-2$

From Equation 2 ... The new wake up rate for $\mathrm{J}$ node is determined which depends on the energy remaining of Node $\mathbf{J}$

4. SIMULATION RESULT

Table 1: Simulation Parameter

\begin{tabular}{|l|l|}
\hline \multicolumn{1}{|c|}{ Parameters } & \multicolumn{1}{c|}{ Values } \\
\hline Number of Nodes & 10 \\
\hline Number of Sink & 1 \\
\hline Placement of Source \& Sink & Static \\
\hline Initial Energy & $18720 \mathrm{~J}$ \\
\hline Receive Power & $62 \mathrm{~mW}$ \\
\hline Transmission Power & $62 \mathrm{~mW}$ \\
\hline
\end{tabular}

In this section provide the simulation and results that provide the balancing of energies of nodes so that the life time of wireless sensor network increases. The simulation proposed system using OMNET ++. To simulate in more realistic way 100 nodes over $1 \mathrm{X} 1 \mathrm{~m}$. area with the asynchronous sleep wake up scheduling and Anycast packet forwarding scheme balancing the energy of sensor nodes by updating the wake up interval of each sensor node. With the help of energy balancing we can increase the life time of wireless sensor network. In the Fig.3 shows the energy level of two sensor nodes by the values we see that the energy level of both nodes is nearly same. As shown in the Fig.3 the energy level of nodes 1 and 2 are nearly same. If we plot the graph of different nodes in the network according to the energy vector of different nodes from that we observe that the energy level of different nodes are nearly same. 


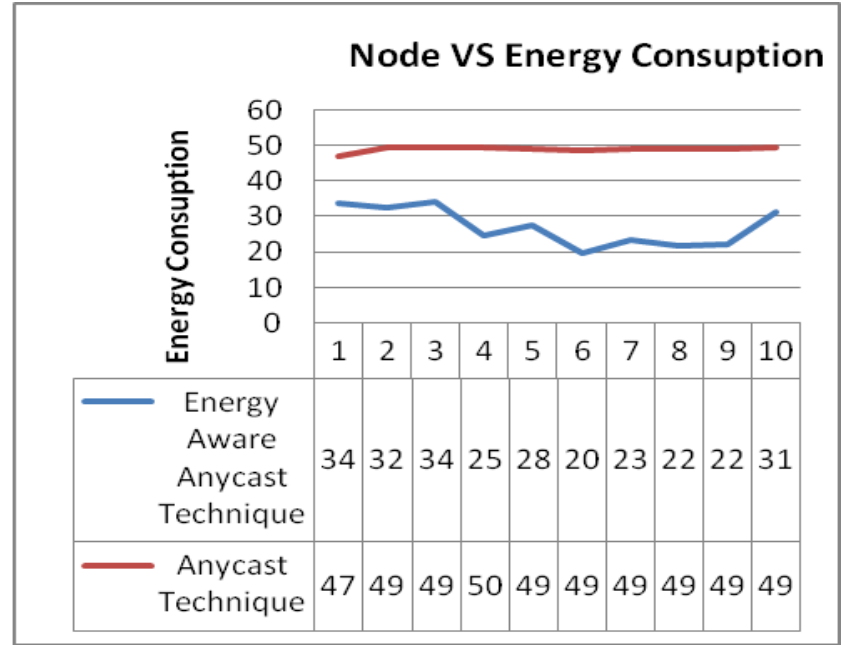

Fig3. Energy Consumed by Sensor Nodes

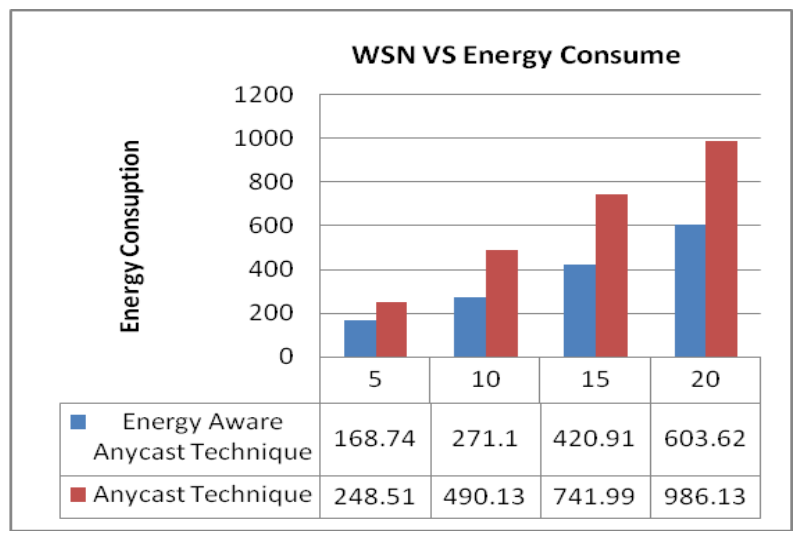

Fig4. Size of WSN VS Energy Consumed

\section{CONCLUSION}

With the help of modification in the wake-up rates of sensor nodes i.e. minimize the wake up rates of nodes having less energy and increase the wake up rates of nodes having higher energy in the asynchronous event driven wireless sensor network we can increase the life time of wireless sensor network.

In this work the main concern is to minimize the energy consumption of wireless sensor node with the help of Anycast packet forwarding technique. In future enhancement the study may be on dynamic adjustment of transmission power and dynamic movement of sensor node to make communication energy enhancement in wireless sensor network.

\section{ADVANTAGES}

Energy aware Anycast technique minimizes the consumption of energy of sensor nodes so that the life time of wireless sensor network.

With the help of Anycast packet forwarding technique minimize the end to end delay when packet is transferred from one sensor node to sink node.

\section{DISADVANTAGES}

In this system Anycast Packet forwarding technique and Asynchronous Sleep Wake up Scheduling is used.
Asynchronous Sleep Wake Up Scheduling brings the end to end delay when packet is transferred .

\section{REFERENCES}

[1] Joohwan Kim, Student Member, IEEE, Xiaojun Lin, Member, IEEE, and Ness B. Shroff, Fellow, "Optimal Anycast Technique for Delay-Sensitive EnergyConstrained Asynchronous Sensor Networks “,IEEE. IEEE/ACM TRANSACTIONS ON NETWORKING, VOL. 19, NO. 2, APRIL 2011

[2] J. Kim, X. Lin, and N. B. Shroff, "Optimal anycast technique for delay sensitive energy-constrained asynchronous sensor networks," in Proc. IEEE INFOCOM, Rio De Janeiro, Brazil, Apr. 2009, pp. 612620

[3] M. Rossi, M. Zorzi, and R. R. Rao, "Statistically assisted routing algorithm (SARA) for hop count based forwarding in wireless sensor networks," Wireless Netw., vol. 14, pp. 55-70, Feb. 2008.

[4] J. Kim, X. Lin, and N. B. Shroff, "Optimal anycast technique for delay-sensitive energy-constrained asynchronous sensor networks, ” Tech.Rep.,2008

[5] M. Rossi and M. Zorzi, "Integrated cost-based MAC and routing techniques for hop count forwarding in wireless sensor networks," IEEE Trans. Mobile Comput., vol. 6, no. 4, pp. 434-448, Apr. 2007.

[6] S. Liu, K.-W. Fan, and P. Sinha, "CMAC: An energy efficient MAC layer protocol using convergent packet forwarding for wireless sensor networks, " in Proc. IEEE SECON, San Diego, CA, Jun. 2007, pp. 11-20.

[7] S. Jain and S. R. Das, "Exploiting path diversity in the link layer in wireless ad hoc networks," in Proc. Jun. 2007, pp. 22-30.

[8] P. Larsson and N. Johansson, "Multiuser diversity forwarding in multihop packet radio networks," in Proc. IEEE WCNC, 2005, vol. 4, pp. 2188-2194.

[9] S. Biswas and R. Morris, "ExOR: Opportunistic multihop routing for wireless networks," in Proc. ACM SIGCOMM, Oct. 2005, vol. 35, pp.133-144.

[10] J. Polastre, J. Hill, P. Levis, J. Zhao, D. Culler, and S. Shenker, "A unifying link abstraction for wireless sensor networks,” in Proc. SenSys,Nov. 2005, pp. 76-89.

[11] Lu, B. Krishnamachari, and C. S. Raghavendra, "An adaptive energy-efficient and low-latency MAC for data gathering in wirelesssensor networks," in Proc. IPDPS, Apr. 2004, pp. 224-231.

[12] W. Ye, H. Heidemann, and D. Estrin, "Medium access control with coordinatedadaptive sleeping for wireless sensor networks," IEEE/ACM Trans. Netw., vol. 12, no. 3, pp. 493-506, Jun. 2004

[13] .J. Polastre, J. Hill, and D. Culler, "Versatile low power media access for wireless sensor networks," in Proc. SenSys, Nov. 2004, pp. 95-107.

[14] T. van Dam and K. Langendoen, "An adaptive energyefficient MAC protocol for wireless sensor networks," in Proc. SenSys, Nov. 2003, pp. 171-180. 